\title{
Regular use of traditional analgesics predicts major coronary events: A cohort study
}

\author{
Mikko Haara' \\ Markku Heliövaara ${ }^{2}$ \\ Jari PA Arokoski ${ }^{3}$ \\ Paul Knekt ${ }^{2}$ \\ Pirjo Manninen ${ }^{4}$ \\ Arpo Aromaa ${ }^{2}$ \\ Antti Reunanen ${ }^{2}$ \\ Olli Impivaara ${ }^{2}$ \\ Heikki Kröger ${ }^{5}$ \\ 'Bone and Cartilage Research Unit \\ (BCRU), University of Kuopio, \\ Kuopio, Finland; ${ }^{2}$ National Public \\ Health Institute, Helsinki and Turku, \\ Finland; ${ }^{3}$ Department of Physical \\ and Rehabilitation Medicine, Kuopio \\ University Hospital, Finland; ${ }^{4}$ Finnish \\ Institute of Occupational Health, \\ Kuopio, Finland; ${ }^{5}$ Department \\ of Surgery/Orthopaedics \\ and Traumatology, Kuopio University \\ Hospital, Kuopio, Finland
}

Correspondence: Mikko Haara Taivaanpankontie 6C 2370200 Kuopio Finland

Tel +3584I 43I 87I6

Email mhaara@hytti.uku.fi

\begin{abstract}
Aims: Serious concern has arisen about the cardiovascular safety of selective cyclo-oxygenase-2 (COX-2) inhibitors. However, recent studies have shown that the cardiovascular risks of regular use of traditional analgesics also deserve attention. We investigated the use of traditional analgesics for their prediction of major coronary events during 16 years of follow-up.

Methods: A population sample of 8000 Finns aged 30 years and over was invited to a comprehensive health examination in 1978-1980; 7217 (90\%) complied, and 4824 of these had no diagnosed cardiovascular disease. The participants filled in a questionnaire eliciting information on the use of analgesics. Record linkage to the National Hospital Discharge Register and the mortality register of the Central Statistical Office of Finland identified 266 major coronary events (myocardial infarctions or coronary deaths) by the end of 1994.

Results: The risk of a major coronary event was significantly elevated among those reporting regular use of analgesics at baseline. Compared with nonusers and adjusted for known risk factors for coronary heart disease, the relative risk of an event during the whole follow-up period was 1.51 (95\% confidence interval [CI] 1.08-2.10) among regular users of analgesics. The risk was as high as 5.27 (95\% CI 2.13-13.11) during the first two years of the follow-up. Thereafter it leveled off.

Conclusion: Based on sales statistics almost all analgesics used in Finland at the end of the 1970's were nonsteroidal antiinflammatory drugs (NSAIDs). Therefore, the increased risk of major coronary events among regular users of analgesics is likely to be due to traditional NSAIDs.
\end{abstract}

Keywords: acute myocardial infarction, coronary heart disease, cohort study, analgesics, pharmacology, risk factors

\section{Background}

New selective nonsteroidal antiinflammatory drugs (NSAIDs) may be prothrombotic and increase the risk of myocardial infarction. Such concerns have arisen after the unexpected findings in a study of gastrointestinal toxicity, indicating higher rates of myocardial infarction in patients receiving a selective cyclo-oxygenase (COX-2) inhibitor (rofecoxib) as compared with those receiving a traditional nonselective NSAID (naproxen). ${ }^{1,2}$ Other recent studies ${ }^{3-5}$ also indicate that the use of COX-2 inhibitors has increased the risk of serious coronary heart disease.

However, traditional NSAIDs also have complex effects that could either prevent or promote coronary heart disease,${ }^{6}$ but despite antiinflammatory and antiplatelet effects similar to those of aspirin these drugs do not protect against myocardial infarction. ${ }^{7}$ Recent results from a large population case-control study show an increased risk of myocardial infarction with current use of rofecoxib, diclofenac, and ibuprofen, even when adjusted for many potential confounders. ${ }^{8}$ Similar results were found in another recent study, in which the relative risk of myocardial infarction was increased in current and new users of all classes of nonaspirin NSAIDs. ${ }^{9}$

Moreover, a recent study suggests that the risk of AMI is increased during several weeks after the cessation of NSAID therapy. ${ }^{10}$ Thus, the cardiovascular safety of all NSAIDs should be reconsidered. 


\section{Aims}

We studied the use of traditional analgesics for their prediction of major coronary events in a large, nationally representative sample of men and women followed-up for 16 years.

\section{Methods}

The study population was a stratified two-stage cluster sample drawn from the population register to represent Finnish adults aged 30 years or over. ${ }^{11}$ In the first stage, 40 representative areas were selected. In the second stage, a systematic sample of inhabitants was drawn from each area. The sample consisted of 8,000 persons $(3,637 \mathrm{men})$ of whom 7,217 (90\%) participated. The study uses material from before current legislation on medical research came into force. Thus, participants were fully informed about the study, they participated in it on a voluntary basis, and the use of the information for medical research was explained to them.

Details of the design and implementation of the MiniFinland Health Survey have been described elsewhere. ${ }^{11,12}$ In brief, all participants were interviewed at home and asked to fill in a basic questionnaire before attending a screening examination. The interview and questionnaire elicited essential information on health habits and previously diagnosed diseases. The screening phase comprised measurements and tests to identify subjects with possible cardiovascular, respiratory or musculoskeletal diseases. The subjects of the present study $(n=4824)$ were those with no cardiovascular disease at the screening phase.

In the basic questionnaire the participants were asked about the use of analgesics: Have you in the past three months taken any medicine (prescribed or other) for any of the following reasons: headache, backache, muscle or joint ache, other ache? If "yes", do you need this medicine continually or nearly so? On the basis of this information the use was classified into none, occasional, or regular.

The basic questionnaire also elicited information on free time physical activity categorised into three classes: low, moderate, and high activity. Smoking history was obtained in a standard interview and categorised as follows: never smoked; ex-smoker; current smoker of cigars, pipe or of fewer than 20 cigarettes a day; and current smoker of 20 cigarettes or more a day. Average weekly consumption of beer, wine and strong beverages during the preceding month were also inquired about. The overall alcohol consumption was then calculated and expressed in grams of ethanol per week. The level of education was considered in three categories based on the number of years of education. Standing height and weight were measured at the screening examination, and body mass index (weight $/$ height ${ }^{2}, \mathrm{~kg} / \mathrm{m}^{2}$ ) was used as a measure of relative weight. Serum cholesterol concentrations were determined from serum samples after 1-3 weeks of storage at $-20^{\circ} \mathrm{C}$ with an auto analyser modification of the Liebermann-Burchard reaction. Systolic and diastolic blood pressures were measured, using long cuffs with $12.5 \mathrm{~cm} \times 40 \mathrm{~cm}$ rubber bags according to standard protocol. Those using insulin or other antidiabetic drugs, and those with clearly elevated fasting blood glucose level (over $130 \mathrm{mg} / 100 \mathrm{ml}$ ) and/or abnormal glucose tolerance and glucosuria, were considered diabetics.

Morbidity and mortality in the cohort have been continuously followed since the baseline examination. A major coronary event was defined as either myocardial infarction or coronary death. Nonfatal cases of myocardial infarction (ICD-8 code 410) were identified by linking the study population to the nationwide hospital discharge register, using the patient's unique personal identification number. ${ }^{13}$ Cases of fatal coronary heart disease (ICD-8 codes 410-414) were identified from death certificates, which were obtained for all the deceased from Statistics Finland. Of multiple events at the individual level, only the first major event was registered. During the follow-up period (from the baseline examination to the end of 1994) 266 major coronary events (coronary deaths or myocardial infarction) occurred among men and women $(n=4824)$ who had not shown cardiovascular disease at baseline.

Statistical Analysis System software (SAS Institute, Cary, NC, USA) was used for statistical analysis. Cross-sectional associations between known risk factors for coronary heart disease and the use of analgesics were analysed with the logistic regression model. The Cox's life-table regression model was used to estimate the associations between the use of analgesics and major coronary event. Potential confounding and effect-modifying factors were also entered into the models. Significance of interaction was tested by entering interaction terms into the models. The $95 \%$ confidence intervals (CIs) of the relative risk estimates and the likelihood ratio statistics (chi-squared values expressed as $\mathrm{P}$ values) were based on the models.

\section{Results}

Of the common factors known to affect the risk of coronary heart disease, high age, female sex, high diastolic pressure, low serum high density lipoprotein (HDL) cholesterol level, low level of education, smoking and low physical activity at leisure were significantly associated with regular use of analgesics at baseline (Table 1). Body mass index, systolic 
Table I Adjusted relative risk (RR) and its $95 \%$ confidence interval $(\mathrm{Cl})$ of the use of analgesics occasionally or over three months by age, total cholesterol, serum HDL, systolic and diastolic blood pressures, body mass index, sex, level of education, smoking, alcohol intake and free time physical activity, and diabetes in Finnish men and women aged 30 years or over without cardiovascular diseases. Logistic regression model. Adjusted by age and sex $(n=4824)$

\begin{tabular}{|c|c|c|c|c|}
\hline Continuous variables* & Mean & SD & $\mathbf{R R}$ & $95 \% \mathrm{Cl}$ \\
\hline Age (years) & 47.1 & 12.6 & 1.45 & $1.33-1.57$ \\
\hline Total cholesterol (mmol/l) & 6.8 & 1.3 & 1.01 & $0.93-1.10$ \\
\hline Serum HDL cholesterol (mmol/l) & 1.7 & 0.4 & 0.91 & $0.83-0.99$ \\
\hline Systolic blood pressure $(\mathrm{mmHg})$ & 139.2 & 20.3 & 0.96 & $0.87-1.06$ \\
\hline Diastolic blood pressure $(\mathrm{mmHg})$ & 92.2 & 9.6 & 1.09 & $0.99-1.19$ \\
\hline Body mass index $\left(\mathrm{kg} / \mathrm{m}^{2}\right)$ & 25.2 & 3.8 & 1.04 & $0.96-1.13$ \\
\hline Class variables & $\begin{array}{l}\text { Analgesics } \\
\text { users(n) }\end{array}$ & $\begin{array}{l}\text { Subjects } \\
\text { examined(n) }\end{array}$ & $\mathbf{R} \mathbf{R}$ & $95 \% \mathrm{Cl}$ \\
\hline \multicolumn{5}{|l|}{ Sex } \\
\hline Male & 204 & 2196 & 1.00 & \\
\hline Female & 390 & 2628 & 1.64 & $1.37-1.97$ \\
\hline \multicolumn{5}{|l|}{ Educational level } \\
\hline$<8$ years & 443 & 3024 & 1.00 & \\
\hline $8-12$ years & 105 & 1130 & 0.70 & $0.56-0.89$ \\
\hline$>12$ years & 46 & 670 & 0.53 & $0.39-0.74$ \\
\hline \multicolumn{5}{|l|}{ Smoking status } \\
\hline Never smoked & 333 & 2664 & 1.00 & \\
\hline Ex-smoker & 111 & 941 & 1.34 & $1.04-1.72$ \\
\hline \multicolumn{5}{|l|}{ Pipe, cigars or } \\
\hline$<20$ cigarettes/day & 100 & 760 & 1.52 & $1.17-1.96$ \\
\hline$\geq 20$ cigarettes/day & 50 & 459 & 1.50 & $1.07-2.12$ \\
\hline \multicolumn{5}{|l|}{$\begin{array}{l}\text { Alcohol intake } \\
\text { (grams of ethanol per week) }\end{array}$} \\
\hline 0 & 307 & 1944 & 1.00 & \\
\hline $1-49$ & 244 & 2294 & 0.85 & $0.70-1.03$ \\
\hline $50-249$ & 15 & 209 & 0.67 & $0.38-1.17$ \\
\hline$\geq 249$ & 28 & 377 & 0.73 & $0.48-1.13$ \\
\hline \multicolumn{5}{|l|}{ Leisure time physical activity } \\
\hline Low & 273 & 1516 & 1.00 & \\
\hline Moderate & 244 & 2438 & 0.55 & $0.46-0.67$ \\
\hline High & 76 & 862 & 0.54 & $0.4 I-0.7 I$ \\
\hline \multicolumn{5}{|l|}{ Diabetes } \\
\hline No & 582 & 4723 & 1.00 & \\
\hline Yes & 12 & 101 & 0.72 & $0.39-1.33$ \\
\hline
\end{tabular}

Note: "For continuous variables the relative risks have been given by an increment of standard deviation (SD).

pressure, alcohol intake and diabetes showed no such association and were therefore not regarded as confounding factors in this study.

After adjustment for the potential confounding factors, the risk of major coronary events was significantly increased among the regular users of analgesics, ie, those who had taken such a medicine daily or almost daily over three months, compared with nonusers (Table 2). The relative risk was five-fold during the first two years of the follow-up, but leveled off thereafter.

Excluding the subjects with chronic inflammatory polyarthritis did not substantially change the relative risk estimates described above. None of the interaction terms between the use of analgesics and the potential confounding factors 
Table 2 The relative risk (RR) and its $95 \%$ confidence interval $(\mathrm{Cl})$ of major coronary event at follow-up in Finnish men and women aged 30 years or over, who have used analgesics, but with no cardiovascular disease at baseline. Cox model

\begin{tabular}{|c|c|c|c|c|c|c|}
\hline \multirow[t]{2}{*}{ Use of analgesics } & \multirow{2}{*}{$\begin{array}{l}\text { Analgesics } \\
\text { users } \\
\text { n }\end{array}$} & \multirow{2}{*}{$\begin{array}{l}\text { Subjects } \\
\text { examined } \\
\text { n }\end{array}$} & \multicolumn{2}{|c|}{ Adjusted by age and sex } & \multicolumn{2}{|c|}{ Complete model ${ }^{*}$} \\
\hline & & & $\mathbf{R R}$ & $95 \% \mathrm{Cl}$ & $\mathbf{R R}$ & $95 \% \mathrm{Cl}$ \\
\hline \multicolumn{7}{|l|}{ The whole follow-up } \\
\hline None & 128 & 2440 & 1.00 & & 1.00 & \\
\hline Occasionally & 88 & 1790 & 1.27 & $0.96-1.67$ & 1.20 & $0.91-1.58$ \\
\hline Regular over 3 months & 51 & 594 & 1.69 & $1.22-2.35$ & 1.51 & $1.08-2.10$ \\
\hline \multicolumn{7}{|l|}{ Follow-up of 2 years } \\
\hline None & 9 & 2440 & 1.00 & & 1.00 & \\
\hline Occasionally & 4 & 1790 & 0.91 & $0.28-3.00$ & 1.04 & $0.31-3.48$ \\
\hline Regular over 3 months & 12 & 594 & 5.22 & $2.18-12.46$ & 5.27 & $2.13-13.11$ \\
\hline \multicolumn{7}{|c|}{ Follow-up from 2 to 16 years } \\
\hline None & 119 & 2408 & 1.00 & & 1.00 & \\
\hline Occasionally & 84 & 1777 & 1.28 & $0.97-1.7 \mid$ & 1.20 & $0.90-1.59$ \\
\hline Regular over 3 months & 39 & 575 & 1.40 & $0.97-2.02$ & 1.23 & $0.85-1.78$ \\
\hline
\end{tabular}

Note: *Adjusted by age, sex, diastolic blood pressure, serum HDL, level of education, smoking, and free time physical activity.

proved statistically significant $(\mathrm{p}=0.5-0.8)$ for prediction of major coronary events during the 16-year follow-up.

\section{Discussion}

In the present study, we found an elevated risk of major coronary events among regular users of analgesics. The risk remained high only during the first two years after the baseline examination. The absence of a longer effect suggests that the use of analgesics probably does not contribute to the development of coronary atherosclerosis but rather triggers acute coronary events. The results suggest that during the past decades a substantial proportion of major coronary events have been attributable to the traditional analgesics.

One of the strengths of our study is that it was based on a large, nationally representative health examination survey of those aged 30 years or over. Moreover, the follow-up period in our study extended up to 16 years, clearly exceeding those in previous studies on the same topic. ${ }^{8,9,14,15}$ The Finnish National Hospital Discharge Register operating since 1967 contains information on hospital admissions and discharges from every hospital in the country. This information includes, along with a personal identification code, the primary and secondary diagnoses according to the International Classification of Diseases. By means of these data we were able to identify those study subjects who had been hospitalised for myocardial infarction since the baseline examination. Because myocardial infarction is a severe disease, practically all patients with this condition are likely to be admitted to hospital for acute care. The diagnosis of myocardial infarction had been correctly registered at the three-digit level in the National Hospital Discharge Register in $84.7 \%$ of the hospitalised patients, when checked against the original hospital documents. ${ }^{13}$ Thus, the discharge register information is sufficiently valid for prediction of myocardial infarction at the national level.

A major limitation of our study is the absence of specific information on the names and amounts of the analgesics used by the subjects at the time of the baseline study. However, the role of analgesics can be estimated on the basis of sales statistics of these drugs. At the time of the baseline study the sales statistics of simple NSAID preparations in Finland was topped by acetylsalicylic acid, ${ }^{16}$ followed by indomethacin, ibuprofen, naproxen, and ketoprofen (Figure 1). The use of paracetamol was very low in Finland at the time of the baseline study, ${ }^{16}$ (Figure 1.) and antimigraine preparations were also of little importance since they were not used on a regular basis. ${ }^{16}$ Most importantly, our results cannot be confounded by selective COX-2 inhibitors, because they were first introduced after the end of the follow-up period. Thus, most of the preparations used regularly for pain relief in this study cohort were likely to be traditional NSAIDs. It appears, therefore, that the increased risk of major coronary events in the present study is associated with regular use of traditional NSAIDs. However, analgesics use was only assessed at baseline which may not necessarily indicate long-term use throughout the follow-up. Furthermore, 


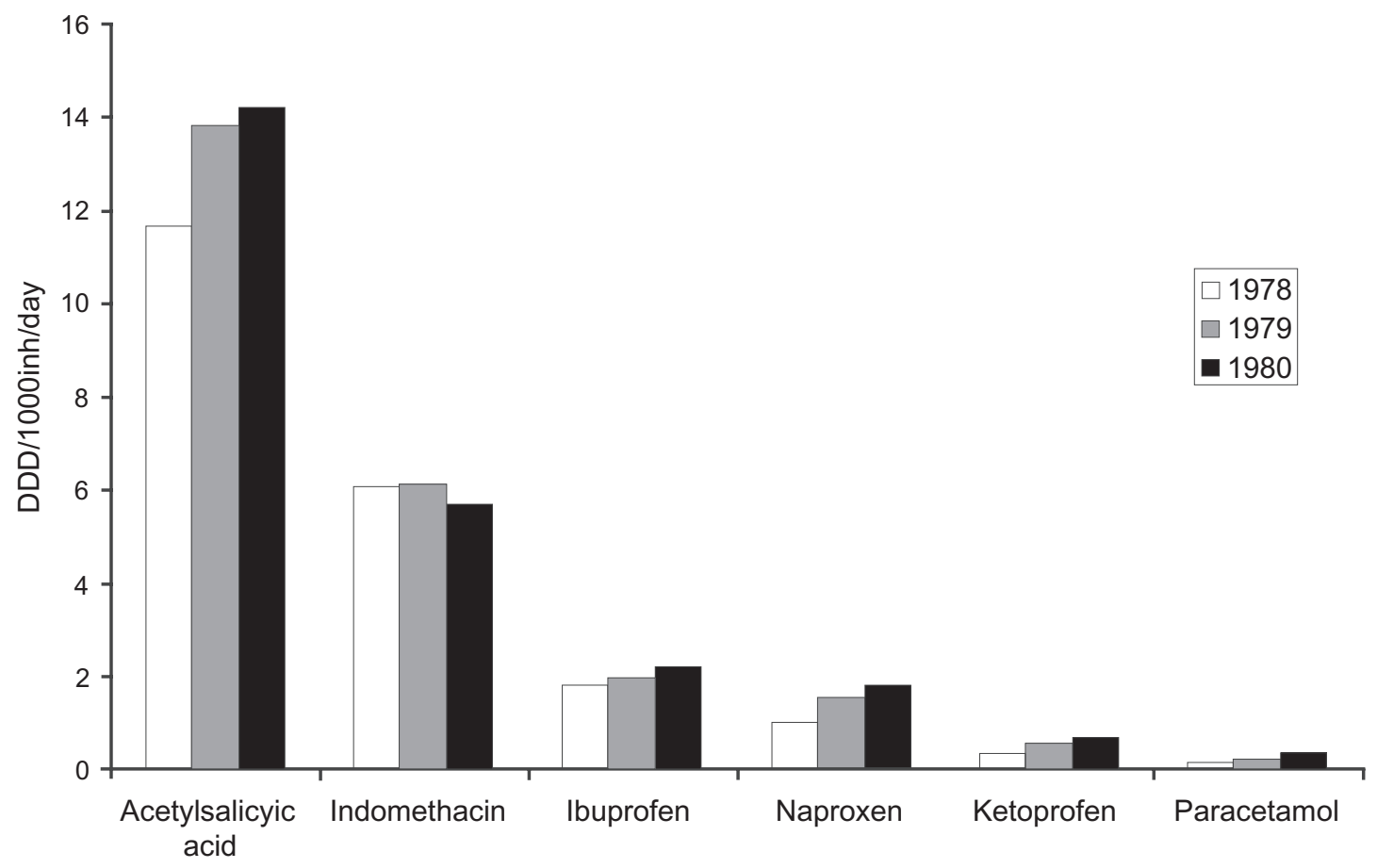

Figure I Sales statistics of simple analgesic preparations in Finland during the baseline study from 1978 to 1980 (excluding sales to hospitals). . $^{*}$

Notes: "The defined daily dose (DDD) is based on the average daily dose used for the main indication of the drug. Drug utilization in DDDs per I000 inhabitants per day indicates the proportion of subjects receiving regular treatment with a particular drug during a given year.

the lack of specific information on the analgesics and their dosing schemes does not allow any conclusion regarding possible causality between specific drugs and cardiovascular risk. Moreover, it has been suggested that concurrent use of acetosalisylic acid should be taken into account because this medicine could mitigate an increased risk of coronary events among some patients using other NSAIDs. ${ }^{17}$ However, our results are unlikely to be confounded by such joint medication because low-dose acetosalisylic acid was not used in Finland at the time of the baseline examination.

An association between NSAIDs and cardiovascular risk was first reported about COX-2 selective preparations. ${ }^{1,3-5}$ Recent studies, however, also linked an increased risk of myocardial infarction with the use of traditional NSAIDs which inhibit both COX-1 and COX-2 receptors. ${ }^{8,9}$ Further, a recent large follow-up study showed an increased risk of myocardial infarction with the use of indomethacin $(+71 \%)$, sulindac (+41\%), and meloxicam $(+37 \%) .{ }^{14}$ Among the COX-2 inhibitors, rofecoxib showed the highest risk $(+32 \%)$, followed by celecoxib $(+9 \%) .{ }^{14}$ The authors suggested that the increased risk for myocardial infarction results from a class effect of NSAIDs. ${ }^{14}$ Similarly, the Finnish population-based matched case-control study showed a modestly increased risk of first myocardial infarction associated with the current use of all NSAIDs. ${ }^{15}$
Moreover, in a prospective cohort of 70,971 women it was found that the risk for major cardiovascular events was associated with the use of NSAIDs or acetaminophen at high frequency or dose, particularly among current smokers. ${ }^{18}$ There was no increased risk with the less than daily use of NSAIDs or acetaminophen. ${ }^{18}$ It is well known that low-dose acetylsalicylic acid (50-250 mg/day) is highly cardioprotective, when it because of irreversible inactivation of platelet COX-1 activity for up to 10 days. ${ }^{19}$ However, there is evidence that high-dose acetylsalicylic acid (over $325 \mathrm{mg} /$ day) can modestly increase the risk of myocardial infarction similarly to other conventional NSAIDs. ${ }^{14,20}$ The high-dose acetylsalicylic acid therapy may even then provide cardioprotective benefits in long term use. ${ }^{21}$ Since acetylsalicylic acid was the most sold analgesic at the baseline of the present study, it is obvious that it was used as a high-dose analgesic, not as a low-dose cardioprotector. The previous studies also demonstrated that the regular use of any NSAIDs with high dose is particularly harmful for patients with known risk factors for coronary heart disease, such as tobacco smoking.

According to a recent meta-analysis, there was an increase of $42 \%$ (relative risk 1,$42 ; 95 \%$ CI $1.13-1.78$ ) in the vascular events, mainly myocardial infarction, among users of selective COX-2 inhibitors, ${ }^{22}$ whereas nonselective NSAIDs 
as a class showed an increase of $19 \%$ (relative risk 1,19 ; CI 1.08-1.31). ${ }^{23}$ Although the overall relative risk appears to be small, the absolute risk may be considerable due to the large number of patients prescribed NSAIDs. ${ }^{24}$

The mechanism behind these associations between NSAID use and cardiovascular risks is partially unknown. Nevertheless, the isozymes, cyclooxygenase-1 (COX-1) and cyclooxygenase-2 (COX-2), are known to catalyze the conversion of arachidonic acid to eicosanoids that play an important role in the maintenance of cardiovascular hemostasis. Thromboxane A2, primarily synthesized by platelet COX-1, causes irreversible platelet aggregation, vasoconstriction and smooth muscle proliferation. In contrast, vascular prostaglandin $\mathrm{I} 2$, which appears to be synthesized by COX-2, counteracts most of the effects exerted by thromboxane A2. Inhibition of the COX isozymes by regular use of traditional NSAIDs or new COX-2 selective inhibitors may therefore disturb the balance between these two routes through which vasoactive eicosanoids are formed. This results in platelet aggregation, vasoconstriction, myocardial infarction and coronary death. ${ }^{25}$ It is also well documented that, most NSAIDs raise blood pressure (approximately $3-5 \mathrm{mmHg}$ ) by inhibiting prostacyclin in the kidney with resultant sodium and water retention, and hypertension. ${ }^{26-28}$ Even such a modest rise in systolic blood pressure can increase the frequency of congestive cardiac failure by $10 \%-20 \%$ and angina by $12 \%{ }^{29}$

\section{Conclusions}

In conclusion, our results support recent observations suggesting that the risk of major coronary events associated with regular use of NSAIDs is not confined to the use of selective COX-2 inhibitors, but also involves traditional NSAIDs. Patient selection rather than drug selectivity may thus be more important, and the lowest possible dose should be used for the shortest possible duration. In the present study, the use of analgesics predicted major coronary events predominantly during the first two years after the baseline examination indicating that the risk of these events is likely to be reversible. Despite the limitations of previous studies and the present one, doctors should be aware of this risk, especially when treating patients with known risk factors for coronary heart disease. Further prospective studies on specific drugs are needed to clarify the effects of these widely prescribed therapies.

\section{Acknowledgments}

This study has been partly supported by the EVO funding of Kuopio University Hospital and the Finnish Orthopaedics
Research Foundation. The authors thank Marianna Sunnari and Jacqueline Välimäki from the University of Turku, Finland, for checking the language of this article. The authors also thank Tinna Voipio from the Finland National Agency of Medicines and Docent Hannu Kokki from the Kuopio University Hospital, Finland for their valuable help concerning the sales statistics of analgesics. The authors declare no conflicts of interest in this work.

\section{References}

1. Bombardier C, Laine L, Reicin A, et al. Comparison of upper gastrointestinal toxicity of rofecoxib and naproxen in patients with rheumatoid arthritis. VIGOR Study Group. N Engl J Med. 2000;343:1520-8.

2. Howes LG, Krum H. Selective cyclo-oxygenase-2 inhibitors and myocardial infarction: how strong is the link? Drug Saf. 2002;25:829-35.

3. Solomon DH, Schneeweiss S, Glynn RJ, et al. Relationship between selective cyclooxygenase-2 inhibitors and acute myocardial infarction in older adults. Circulation. 2004;109:2068-73.

4. Graham DJ, Campen D, Hui R, et al. Risk of acute myocardial infarction and sudden cardiac death in patients treated with cyclo-oxygenase 2 selective and non-selective non-steroidal anti-inflammatory drugs: nested case-control study. Lancet. 2005;365:475-81.

5. Levesque LE, Brophy JM, Zhang B. The risk for myocardial infarction with cyclooxygenase-2 inhibitors: a population study of elderly adults. Ann Intern Med. 2005;142:481-9.

6. Ray WA, Stein CM, Hall K, et al. Non-steroidal anti-inflammatory drugs and risk of serious coronary heart disease: an observational cohort study. Lancet. 2002;359:118-23.

7. Solomon DH, Glynn RJ, Levin R, et al. Nonsteroidal anti-inflammatory drug use and acute myocardial infarction. Arch Intern Med. 2002;162:1099-104.

8. Hippisley-Cox J, Coupland C. Risk of myocardial infarction in patients taking cyclo-oxygenase-2 inhibitors or conventional non-steroidal antiinflammatory drugs: population based nested case-control analysis. BMJ. 2005;330:1059-63.

9. Johnsen SP, Larsson H, Tarone RE, et al. Risk of hospitalization for myocardial infarction among users of rofecoxib, celecoxib, and other NSAIDs: a population-based case-control study. Arch Intern Med. 2005; 165:978-84.

10. Fischer LM, Schlienger RG, Matter CM, et al. Current use of nonsteroidal anti-inflammatory drugs and the risk of acute myocardial infarction. Pharmacotherapy. 2005;25:503-10.

11. Aromaa A, Heliövaara M, Impivaara $\mathrm{O}$, et al. Health, functional limitations and need for care in Finland. Basic results from the Mini-Finland Health Survey. Helsinki and Turku: Publications of the Social Insurance Institution; 1989.

12. Haara MM, Manninen P, Kröger H, et al. Osteoarthritis of finger joints in Finns aged 30 or over: prevalence, determinants, and association with mortality. Ann Rheum Dis. 2003;62:151-8.

13. Heliövaara M, Reunanen A, Aromaa A, et al. Validity of hospital discharge data in a prospective epidemiological study on stroke and myocardial infarction. Acta Med Scand. 1984;216:309-15.

14. Singh G, Mithal A, Triadafilopoulos G. Both selective COX-2 inhibitors and non-selective NSAIDs increased the risk of acute myocardial infarction in patients with arthritis: Selectivity is with the patient, not the drug class. Presented at The Annual European Congress of Rheumatology of the European League against Rheumatism (EULAR); June 8-11, 2005, Vienna, Austria.

15. Helin-Salmivaara A, Virtanen A, Vesalainen R, et al. NSAID use and the risk of hospitalization for first myocardial infarction in the general population: a nationwide case-control study from Finland. Eur Heart J. 2006;27:1657-63. 
16. Nordic Statistic on medicines 1978-1980. Uppsala: Nordic Council on Medicines;1982; p. 133-47.

17. Antman EM, Bennett JS, Daugherty A, et al. American Heart Association. Use of nonsteroidal antiinflammatory drugs an update for clinicians - a science advisory from the American Heart Association. Circulation. 2007;115:1634-42.

18. Chan AT, Manson JE, Albert CM, et al. Nonsteroidal anti-inflammatory drugs, acetaminophen, and the risk of cardiovascular events Circulation. 2006;113:1578-87.

19. Hermann M, Krum H, Ruschitzka F. Editorial: To the Heart of the Matter. Coxibs, smoking and cardiovascular risk. Circulation. 2005;112:941-5.

20. Taylor DW, Barnett HJ, Ferguson GG, et al. Low-dose and high-dose acetylsalicylic acid for patient undergoing carotid endarterectomy: a randomized controlled trial. Lancet. 1999;353:2179-84.

21. Antithrombotic Trialists Collaboration. Collaborative meta-analysis of randomised trials of antiplatelet therapy for prevention of death, myocardial infarction, and stroke in high risk patients. BMJ. 2002;324:71-86.

22. Kerney PM, Baignent C, Godwin J, et al. Do selective cyclooxygenase- 2 inhibitors and traditional non-steroidal anti-inflammatory drugs increase the risk of atherostrombosis? Meta-analysis of randomised trials. BMJ. 2006;332:1302-8.
23. Singh $\mathrm{G}, \mathrm{Wu} \mathrm{O}$, Langhorne $\mathrm{P}$, et al. Risk of acute myocardial infraction with non-selective non-steroidal anti-inflammatory drugs: a metaanalysis. Arthritis Res Ther. 2006;8:R153.

24. Madhok R, Wu O, McKellar G, et al. Non-steroidal anti-inflammatory drugs-changes in prescribing may be warranted. Rheumatology. 2006;45:1458-60.

25. Garcia Rodriguez LA. The effect of NSAIDs on the risk of coronary heart disease: fusion of clinical pharmacology and pharmacoepidemiologic data. Clin Exp Rheumatol. 2001;19(6 Suppl 25):S41-4.

26. Johnson AG, Nguyen TV, Day RO. Do non-steroidal anti-inflammatory drugs affect blood pressure? A meta-analysis. Ann Intern Med. 1994;121:289-300.

27. Aisen PS, Schafer K, Grundman M, et al. NSAIDs and hypertension. Arch Intern Med. 2003;163:1115.

28. Grosser T, Fries S, FitzGerald GA. Biological basis for the cardiovascular consequences of COX-2 inhibition: therapeutic challenges and opportunies. J Clin Invest. 2006;116:4-15.

29. ALLHAT Collaborative Research Group. Major cardiovascular events in hypertensive patients randomized to doxazosin vs chlorthalidone. JAMA. 2000;283:1967-75. 
